\title{
Perspectives
}

\section{Whither must spermatozoa wander? The future of laboratory seminology}

\author{
R. John Aitken
}

ARC Centre of Excellence in Biotechnology and Development, Discipline of Biological Sciences, University of Newcastle, Callaghan NSW 2308, Australia

\begin{abstract}
This commentary celebrates the publication of the 5th edition of the World Health Organization Laboratory Manual for the Examination and Processing of Human Semen. This is the most complete text to date on the creation of a conventional semen profile and includes invaluable reference limits for specific aspects of semen quality based on the analysis of over 1900 recent fathers. The new edition of the manual also includes detailed protocols for monitoring different aspects of sperm function and new chapters on the preparation of spermatozoa for assisted conception and cryopreservation. Given that this publication is the definitive statement on how to perform a descriptive semen analysis, we might speculate on the future of this field and the sorts of tests that might feature in future editions of the manual. Cell biologists are currently being empowered by the 'omics revolution, which is placing at their disposal technologies of unprecedented power to examine the biochemical composition of cells such as spermatozoa. Indeed, spermatozoa are perfect vehicles for this kind of analysis because they can be obtained as extremely pure suspensions, exist naturally in isolation and can be induced to express their capacity for fertilization and the initiation of embryonic development in vitro. The application of 'omics technologies to these cells, in concert with detailed assessments of their functional competence, should provide insights into the biochemical basis of defective semen quality. This information will then help us understand the causes of male infertility and to develop rational methods for its treatment and possible prevention.
\end{abstract}

Asian Journal of Andrology (2010) 12: 99-103. doi: 10.1038/aja.2008.42

Keywords: assisted conception, DNA damage, male infertility, miscarriage, semen, seminology, spermatozoa

\section{In the beginning}

A long long time ago, when in vitro fertilization (IVF) was in its first flush of youth and Intracytoplasmic sperm injection (ICSI) had not driven seminology into the reproductive wilderness, the development of robust methods for the diagnosis and treatment of male infertility was regarded as a noble cause worthy of engagement.

Correspondence to: Prof. R. John Aitken, Laureate Professor of Biological Sciences, Discipline of Biological Sciences, School of Environmental and Life Sciences, University of Newcastle, Callaghan, NSW 2308, Australia.

Fax:+61-2-4921-6308_E-mail: jaitken@mail.newcastle.edu.au

Received: 3 November 2008 Revised: 4 November 2008

Accepted: 6 November 2008
In those far-off days, the descriptive semen profile was the only means we had of determining the fertility of men attending infertility clinics. This profile focused on an analysis of sperm number, motility and morphology underpinned by the fundamental belief that fertility is essentially a war of attrition. According to this model, the ejaculate must contain more than a certain critical number of motile, morphologically normal spermatozoa in order to withstand the cellular carnage that inevitably accompanies the perilous transition from the point of insemination to the site of fertilization. In diagnostic terms, the concept that male fertility is essentially 'a numbers game' resulted in a preoccupation with threshold counts for sperm number, motility and morphology that define the conventional semen profile, even to this day. The belief that fertility is entirely dependent on sperm number was, ironically, 
dispelled by experiments designed to develop hormonal methods of male contraception. In these studies, a small proportion of normal men treated with exogenous steroids experienced a dramatic decline in sperm concentrations well into the pathological range $\left(\leq 3\right.$ million $\left.\mathrm{mL}^{-1}\right)$ and yet could still impregnate their partners, although it might have taken some time $[1,2]$. A similar situation pertains when men experiencing a lack of gonadotrophic drive to the testes because of hypogonadotrophic hypogonadism are treated in adulthood with gonadotrophins or pulsatile gonadotrophin-releasing hormone [3]. As these men have small testes, they generate low numbers of spermatozoa. However, because the only deficiency in these men is a lack of gonadotrophins, endocrine replacement therapy initiates a spermatogenic process that is fundamentally normal. As a result, spermatozoa generated under these circumstances are functional and, given sufficient time, can eventually initiate pregnancies although the concentration of spermatozoa in the ejaculate would have been considered pathological in conventional terms [3, 4]. Such data simply serve to emphasize that there is more to the definition of fertility than sperm number. The conventional semen profile is of diagnostic significance because it reflects the quality of the underlying spermatogenic process, and it is the latter that determines the functional competence of the spermatozoa and the fertilizing potential of the ejaculate.

In many ways, if the quality of spermatogenesis is the target of our analysis, the conventional semen profile, with its emphasis on sperm concentration, is possibly not the best way to make such an assessment. Sperm concentration is highly dependent on ejaculate volume, which, in turn, has as much to do with the frequency of ejaculation as the quality of spermatogenesis. It was once suggested to me by Rune Eliasson that a more valid read-out of the quality of spermatogenesis might be the total sperm count divided by the testicular volume as determined by an orchidometer. Although this seems like an eminently sensible suggestion, the conventional criteria of semen quality have become so well entrenched in routine laboratory practice that it is probably too late to make a change.

If we are stuck with the conventional semen profile, then it is incumbent on us to ensure that such analyses are performed carefully, according to a universally agreedupon set of guidelines using standard operating procedures. When I first entered the andrological arena 30 years ago, there was no real consensus in this area and quality control was a distant glint in David Mortimer's eye. An important element of order and reason was brought to this chaotic situation through successive revisions of the World Health Organization (WHO) semen manual. It is staggering how many methodological variations can exist for something as simple as a sperm count, but this is a reality in this type of science. When it comes to something as complex as sperm morphology or as dynamic as sperm motility, the difficulties encountered in delivering high-quality, robust data are magnified. Addressing such methodological issues is critical because only through such means can we hope to generate reliable information on semen quality that can be accurately compared across different laboratories. Once a high level of standardization has been achieved, it will be possible to mount international studies on changes in semen quality in relation to such factors as workplace exposure, geographical location or even the passage of time. Similarly, standardized robust protocols will be necessary if we are to generate comparable data from different centres on the effectiveness of new approaches to male contraception.

This edition of the Asian Journal of Andrology is celebrating the latest, and certainly the most extensive, edition of the WHO manual to date. Fittingly, this manual is dedicated to Geoffrey Waites, former manager of the WHO Male Task Force and a pioneer in the application of rigorous scientific principles to the study of male reproduction. This version of the manual is also a testament to the editorial rigour, dedication, sagacity, wisdom, persistence and enthusiasm of one of Geoff's protégés, the Editor-in-Chief Trevor Cooper. This new version of the manual has been dramatically improved relative to all previous versions and contains a wealth of methodological detail about how to construct the various elements of the conventional semen profile, as well as new chapters on sperm preparation for assisted conception and cryopreservation. The text also incorporates a series of detailed standardized protocols for undertaking more advanced assessments of semen quality, including the detection of leukocytes, the identification of precursor germ cells and the determination of anti-sperm antibodies. Most importantly, there is a completely revised chapter on quality control and, for the first time, data supporting reference limits. The latter were acquired by analysing semen samples from 1800 recent fathers (time-topregnancy of $\leq 12$ months) living in eight countries on three continents. From these data, one-sided lower reference limits were generated based on the 5th percentile of the data distribution. These values amount to longneeded evidence-based thresholds that should be a considerable benefit to patients and clinicians alike in calculating the relative fertility of a given donor. Intriguingly, the values generated via this process are not a million miles away from the existing thresholds of normality for the conventional semen profile.

Notwithstanding the considerable importance of this manual for the standardized creation and interpretation of semen profile data, this form of laboratory assessment is 
not (as Churchill would have said) 'the end, not even the beginning of the end, but simply the end of the beginning' as far as the diagnosis of male infertility is concerned. It should be possible to add value to this fundamental form of analysis by conducting direct tests of sperm function, rather than indirectly inferring this property from the semen profile. In order to facilitate such functional analyses, the new manual has also incorporated a series of standardized protocols for undertaking more advanced evaluations of semen quality, including assessments of cervical mucus penetration, zona binding assays, techniques for measuring the acrosome reaction, the Hamster oocyte penetration test and computer-assisted sperm analysis (CASA) analysis of sperm movement.

Despite the evident value of these protocols to gamete biologists with an intense interest in sperm biology, it is also important to recognize that ICSI is now a major therapeutic option for male infertility, for which assessments of sperm function are largely irrelevant. However, for this form of therapy, analysis of sperm chromatin integrity has become extremely important in light of the wealth of circumstantial evidence linking DNA damage in human spermatozoa with adverse clinical outcomes, including poor fertilization rates, impaired embryonic development, an increased risk of miscarriage and morbidity in the offspring, including childhood cancer [5-8]. The manual touches on this area but does not develop it. The lack of attention to this area is partly due to the fact that the manual has taken 3.5 years to produce, from the initial gathering of the working group in Geneva to the date of publication. In the interim, a great deal of data have been generated on the nature and significance of DNA damage in the male germ line that was not available when the text was being prepared. This is clearly an area of diagnostic seminology that must be developed further in future editions of the manual.

\section{The future}

So, what of the future? In terms of basic seminology, the 5th edition of the WHO manual may prove to be a definitive account of how such assessments should be performed. I also doubt whether future editions will add a great deal to the functional assay section of the manual because the prevalence of ICSI as a therapeutic procedure has dampened clinical enthusiasm for detailed, expensive and time-consuming assessments of fertilizing potential. However, I do believe the functional assays described in the manual are fundamental to the future of diagnostic andrology. This is not because these assays have diagnostic utility in their own right (even if some do), but rather because they serve as valuable research tools that will allow researchers to investigate the molecular basis of defective sperm function. On the basis of this information, insights into the aetiology of male infertility should be generated.

The information generated by conventional semen analysis allows the classification of patients into descriptive groups such as oligozoospermic, asthenozoospermic and so on. However, we should never confuse such descriptive categories with our ultimate goal, which is a diagnosis. A single condition such oligozoospermia may involve a multitude of different aetiologies. It is not until we resolve the causes of male infertility at a molecular level that we shall really be able to achieve the holy trinity of diagnosis, treatment and prevention.

In the 21 st century, molecular andrologists will have an ever more powerful range of investigative 'omics with which to examine the defective spermatozoa of infertile patients and gain a deeper understanding of the underlying aetiology. This 'omics revolution has already begun, as shown by the recent publication of a detailed proteomic analysis of human spermatozoa [9], together with initial attempts to determine how the protein structure of normal and defective spermatozoa compare [10-12]. However, such comparisons are meaningless unless the functional defects in the spermatozoa have been precisely defined $[13,14]$. It is only when we are in possession of such functional information that meaningful analyses of structure-function relationships can be undertaken. In this context, the protocols provided by the manual for assessing the functional competence of human spermatozoa will prove an invaluable partner to the biochemical data generated by the new generation of proteomic technologies.

Similarly, metabolomic analyses will help us pinpoint the metabolic defects in these cells that might be associated with impaired function or DNA damage. In this context, oxidative stress is increasingly recognized as a major causative factor in the aetiology of male infertility [15-18]. However, we have only just begun to understand the origins of such stress and the central role played by the mitochondria in the generation of reactive oxygen species by the male gamete [19]. The importance of such insights lies in the opportunities afforded for therapeutic intervention. If oxidative stress is part of the cause of male infertility, then antioxidant therapy must surely be part of the cure. This concept has been in the literature since the 1940s, when $\alpha$-tocopherol was first shown to be an essential vitamin for the preservation of male fertility in rats. Unfortunately, in the interim, no definitive antioxidant trials have been conducted on patients to support this concept. One of the major reasons for this lack of progress lies in the lack of robust methods for measuring oxidative stress in the male germ line. However, new sensitive flow cytometry methods for measuring lipid peroxidation and oxidative DNA damage 
have been developed, which are of direct relevance to this objective $[20,21]$. Novel, robust criteria for assessing the causes or consequences of oxidative stress in the male germ line may also become apparent when lipidomics are applied to the analysis of human sperm quality [22, 23]. Furthermore, glycomic analyses might help resolve the causes of defective sperm-zona interaction [14]. Modern advances in diagnostic genomics might also help us to identify genotypes associated with specific defects in semen quality $[24,25]$. Such information will be an invaluable aid to the diagnosis of certain forms of male infertility and facilitate informed decision making by the couple.

The role the spermatozoon may play in the origins of genetic mutations may also be a feature of diagnostic andrology in the future. It has been clear for many decades that most, if not all, dominant genetic diseases originate in the male germ line and are highly correlated with male age [26]. Although the aetiology of these diseases has traditionally been laid at the door of replication error, this hypothesis does not account for all of the facts, particularly in the case of achondroplasia [27, 28]. As an alternative explanation, we have suggested that such mutations arise as a consequence of the aberrant repair of sperm DNA by the oocyte between the moment of fertilization and S-phase of the first mitotic division [16, 29]. In these circumstances, DNA damage in spermatozoa can be seen as a form of pro-mutation that inadvertently becomes converted into a mutation by the egg in a flawed attempt at DNA repair. Such an explanation might also account for the wide range of polygenic neurological disorders (epilepsy, bipolar disease, spontaneous schizophrenia, autism) that occur in children and that are correlated with paternal age at the moment of conception $[5,6,30$ 32]. This model might also explain the childhood cancer associated with DNA damage induced in spermatozoa by the fathers' heavy smoking [33, 34]. In light of these considerations, detailed examination of DNA damage in human spermatozoa would certainly be an important objective for future versions of the WHO manual. At present, we have an array of assays (including Comet, TUNEL, SCSA and the sperm chromatin dispersal test), but no clear indication as to which test is optimal, why it is optimal and which protocol should be used in its execution. Future versions of the WHO manual must address these critical issues.

It should also be recognized that DNA damage may not simply be a matter of base adducts or strand breakage. Several studies have emphasized the presence of epigenetic defects in spermatozoa that could have an impact on the developmental normality of the developing embryo. Such epigenetic factors include (i) a functional centrosome to regulate cell division in the embryo; (ii) an appropriate pattern of chromatin remodelling, including the presence of protamines 1 and 2 in the ratio $1: 1$, and, in the case of human spermatozoa, suitably modified histones; (iii) an appropriate population of mRNA and miRNA species that will be transferred to the zygote by the fertilizing spermatozoon and may play a key role in the regulation of early embryonic development; and (iv) an appropriate pattern of DNA methylation. Several recent papers indicate that the DNA methylation profile is dramatically altered in the spermatozoa of infertile men [35-37]. Moreover, we already know that the incidence of imprinting defects such as Angelman syndrome (AS) and Beckwith-Weidemann syndrome (BWS) is elevated in children born as a result of assisted conception [38]. Assessment of such factors is likely to be a key issue for the 6th edition of the WHO manual as we move the consideration of normal sperm function beyond the traditional realms of 'fertilizing potential' and into the factors responsible for the initiation of normal embryonic development.

Hopefully, future editions of the WHO manual will also contain protocols for measuring aspects of semen quality that are not only diagnostically significant, but also therapeutically instructive in that they are linked to strategies for either the prevention or the correction of the underlying pathology. In a perfect world, consensus protocols for the treatment of the infertile male, such as optimized antioxidant regimes, will ultimately find their way into the manual. In this way, robust methods for the diagnosis and management of male infertility could be effectively linked within a single reference volume.

Clearly, laboratory seminology is still very much in its infancy. Inasmuch as the creation of a conventional semen profile will always represent the foundations of male fertility evaluation, the 5th edition of the WHO manual is a definitive statement on how such assessments should be carried out and how the quality should be controlled. It is to be hoped that andrologists will read the text carefully and adopt the protocols with such enthusiasm and precision that data sets from different laboratories will be directly comparable. However, as diagnostic laboratory andrology develops as a discipline, future editions of the WHO manual will inevitably move beyond the provision of consensus protocols for the conventional semen profile and into the assessment of biochemical criteria, which will shed light on the underlying pathophysiology of the infertile condition and suggest strategies for its effective management and prevention.

\section{References}

1 Barfield A, Melo J, Coutinho E, Alvarez-Sanchez F, Faundes A, et al. Pregnancies associated with sperm concentrations 
below 10 million $/ \mathrm{mL}$ in clinical studies of a potential male contraceptive method, monthly depot medroxyprogesterone acetate and testosterone esters. Contraception 1979; 20: 121-7.

2 Wallace EM, Aitken RJ, Wu FC. Residual sperm function in oligozoospermia induced by testosterone enanthate administered as a potential steroid male contraceptive. Int J Androl 1992; 15: 416-24.

3 Kliesch S, Behre HM, Nieschlag E. High efficacy of gonadotropin or pulsatile gonadotropin-releasing hormone treatment in hypogonadotropic hypogonadal men. Eur J Endocrinol 1994; 131: 347-54.

4 Liu PY, Gebski VJ, Turner L, Conway AJ, Wishart SM, et al. Predicting pregnancy and spermatogenesis by survival analysis during gonadotrophin treatment of gonadotrophin-deficient infertile men. Hum Reprod 2002; 17: 625-33.

5 Aitken RJ, De Iuliis GN. Origins and consequences of DNA damage in male germ cells. Reprod Biomed Online 2007; 14: 727-33.

6 Aitken RJ, De Iuliis GN. Value of DNA integrity assays for fertility evaluation. Soc Reprod Fertil Suppl 2007; 65: 81-92.

7 Lewis SE, Aitken RJ. DNA damage to spermatozoa has impacts on fertilization and pregnancy. Cell Tissue Res 2005; 322: 33-41.

8 Lewis SE, Agbaje I, Alvarez J. Sperm DNA tests as useful adjuncts to semen analysis. Syst Biol Reprod Med 2008; 54: 111-25.

9 Baker MA, Reeves G, Hetherington L, Müller J, Baur I, et al. Identification of gene products present in Triton X-100 soluble and insoluble fractions of human spermatozoa lysates using LCMS/MS analysis. Proteomics Clin Appl 2007; 1: 524-32.

10 Aitken RJ, Baker MA. The role of proteomics in understanding sperm cell biology. Int J Androl 2008; 31: 295-302.

11 Barratt CL. The human sperm proteome: the potential for new biomarkers of male fertility and a transformation in our understanding of the spermatozoon as a machine: commentary on the article 'Identification of proteomic differences in asthenozoospermic sperm samples' by Martinez et al. Hum Reprod 2008; 23: 1240-1.

12 Martínez-Heredia $\mathrm{J}$, de Mateo $\mathrm{S}$, Vidal-Taboada JM, Ballescà $\mathrm{JL}$, Oliva R, et al. Identification of proteomic differences in asthenozoospermic sperm samples. Hum Reprod 2008; 23: 783-91.

13 Liu DY, Baker HW. A new test for the assessment of spermzona pellucida penetration: relationship with results of other sperm tests and fertilization in vitro. Hum Reprod 1994; 9: 489-96.

14 Liu DY, Baker HW. High frequency of defective sperm-zona pellucida interaction in oligozoospermic infertile men. Hum Reprod 2004; 19: 228-33.

15 Aitken RJ. The role of free oxygen radicals and sperm function. Int J Androl 1989; 12: 95-7.

16 Aitken RJ, Krausz C. Oxidative stress, DNA damage and the Y chromosome. Reproduction 2001; 122: 497-506.

17 Tremellen K. Oxidative stress and male infertility - a clinical perspective. Hum Reprod Update 2008; 14: 243-58.

18 Turner TT, Lysiak JJ. Oxidative stress: a common factor in testicular dysfunction. J Androl 2008; 29: 488-98.

19 Koppers AJ, De Iuliis GN, Finnie JM, McLaughlin EA, Aitken RJ, et al. Significance of mitochondrial reactive oxygen species in the generation of oxidative stress in spermatozoa. J Clin
Endocrinol Metab 2008; 93: 3199-207.

20 Hwang ES, Bowen PE. DNA damage, a biomarker of carcinogenesis: its measurement and modulation by diet and environment. Crit Rev Food Sci Nutr 2007; 47: 27-50.

21 Aitken RJ, Wingate JK, De Iuliis GN, McLaughlin EA. Analysis of lipid peroxidation in human spermatozoa using BODIPY C11. Mol Hum Reprod 2007; 13: 203-11.

22 Aitken RJ, Wingate JK, De Iuliis G, Koppers AJ, McLaughlin EA, et al. Cis-unsaturated fatty acids stimulate reactive oxygen species generation and lipid peroxidation in human spermatozoa. J Clin Endocrinol Metab 2006; 91: 4154-63.

23 Jones R, Mann T, Sherins R. Peroxidative breakdown of phospholipids in human spermatozoa, spermicidal properties of fatty acid peroxides, and protective action of seminal plasma. Fertil Steril 1979; 31: 531-7.

24 Cram D, Lynch M, O'Bryan MK, Salvado C, McLachlan RI, et al. Genetic screening of infertile men. Reprod Fertil Dev 2004; 16 : 573-80.

25 O’Bryan MK, de Kretser D. Mouse models for genes involved in impaired spermatogenesis. Int J Androl 2006; 29: 76-89.

26 Crow JF. The origins, patterns and implications of human spontaneous mutation. Nat Rev Genet 2000; 1: 40-7.

27 Hurst LD, Ellegren H. Human genetics: mystery of the mutagenic male. Nature 2002; 420: 365-6.

28 Tiemann-Boege I, Navidi W, Grewal R, Cohn D, Eskenazi B, et al. The observed human sperm mutation frequency cannot explain the achondroplasia paternal age effect. Proc Natl Acad Sci USA 2002; 99: 14952-7.

29 Aitken RJ, Koopman P, Lewis SE. Seeds of concern. Nature 2004; 432: 48-52.

30 Sipos A, Rasmussen F, Harrison G, Tynelius P, Lewis G, et al. Paternal age and schizophrenia: a population based cohort study. BMJ 2004; 329: 1070.

31 Reichenberg A, Gross R, Weiser M, Bresnahan M, Silverman $\mathrm{J}$, et al. Advancing paternal age and autism. Arch Gen Psych 2006; 63: 1026-32.

32 Frans EM, Sandin S, Reichenberg A, Lichtenstein P, Långström $\mathrm{N}$, et al. Advancing paternal age and bipolar disorder. Arch Gen Psych 2008; 65: 1034-40.

33 Ji BT, Shu XO, Linet MS, Zheng W, Wacholder S, et al. Paternal cigarette smoking and the risk of childhood cancer among offspring of nonsmoking mothers. J Natl Cancer Inst 1997; 89: 238-44.

34 Lee KM, Ward MH, Han S, Ahn HS, Kang HJ, et al. Paternal smoking, genetic polymorphisms in CYP1A1 and childhood leukemia risk. Leuk Res 2009; 33: 250-8.

35 Aitken RJ. Just how safe is assisted reproductive technology for treating male factor infertility? Exp Rev Obstet Gynecol 2008; 3: 267-71.

36 Carrell DT. Paternal genetic and epigenetic influences on IVF outcome. Exp Rev Obstet Gynecol 2008; 3: 359-67.

37 Houshdaran S, Cortessis VK, Siegmund K, Yang A, Laird PW, et al. Widespread epigenetic abnormalities suggest a broad DNA methylation erasure defect in abnormal human sperm. PLoS ONE 2007; 2: e1289.

38 Li T, Vu TH, Ulaner GA, Littman E, Ling JQ, et al. IVF results in de novo DNA methylation and histone methylation at an Igf2-H19 imprinting epigenetic switch. Mol Hum Reprod 2005; 11: 631-40. 